\title{
Some Observations on the Proof Theory of Second Order Propositional Multiplicative Linear Logic (Extended Abstract)
}

\author{
Lutz Straßburger \\ INRIA Futurs, Projet Parsifal \\ École Polytechnique - LIX — Rue de Saclay — 91128 Palaiseau Cedex — France \\ http://www.lix.polytechnique.fr/ lutz
}

\begin{abstract}
We present two new aspects of the proof theory of MLL2. First, we will give a novel proof system in the framework of the calculus of structures. The main feature of the new system is the consequent use of deep inference. Due to the new freedom of permuting inference rules, we are able to observe a decomposition theorem, which is not visible in the sequent calculus. Second, we show a new notion of (boxfree) proof nets which is inspired by the deep inference proof system. Nonetheless, the proof nets are independent from the deductive system. We have "sequentialisation" into the calculus of structures as well as into the sequent calculus. We present a notion of cut elimination which is terminating and confluent, and thus gives us a category of proof nets.
\end{abstract}

\section{Introduction}

Second order propositional multiplicative linear logic is the fragment of linear logic that contains the multiplicative connectives and second order quantifiers, i.e., one can quantify over propositional variables. We will denote the logic by MLL2, following the nomenclature of [15], where its undecidability was shown. The logic has first been investigated by Girard in [10] where a deductive system in the sequent calculus was given together with a proof of cut elimination and a notion of proof nets.

Girard's proof nets for MLL2 are tightly tied to the sequent calculus: there is a one-to-one correspondence between the inference rule applications in a given sequent proof and the "links" in the corresponding proof net. This can be seen as an advantage because it makes it easy to recover the deductive tree structure from the proof net. However, it can also be seen as a rather unfortunate fact because the sequent calculus is not the only framework for presenting logical systems (although it has a monopoly), and ideally, proof nets should capture the essence of a proof inde- pendently from any formal deductive system. Furthermore, the close connection to the sequent calculus causes certain design flaws in Girard's proof nets, namely, it necessitates the concept of boxes in order to protect subproofs.

In [21, 17], it was shown how boxes for the multiplicative units can be avoided by entirely dropping the connection between nodes in the proof net and rules in the sequent calculus. Here, these ideas are pushed further to accommodate the quantifiers. We consider here only second order propositional quantifiers because the first order case has a rather weak proof theory in multiplicative linear logic (see [2] for a detailed treatment). But with second order quantifiers, the logic becomes already undecidable 1

This work is motivated by the question "What constitutes a proof with quantifiers?" I.e., what is the essential data structure that remains if we abstract away from the deductive systems?

To make some progress towards an answer, we first introduce a new deductive system for MLL2, which will not be given in the sequent calculus, but in the calculus of structures [11 12 5]. Then we present a new notion of proof nets without boxes for MLL2, which comes with a cut elimination procedure which is confluent and terminating. Hence, we get a well-defined category of proofs for MLL2.

We will also show, how the three different presentations of proofs, sequent calculus, calculus of structures, and proof nets, are translated into each other.

\section{MLL2 in the sequent calculus}

Before we start, let us recall how MLL2 is presented in the sequent calculus. Let $\mathscr{A}=\{a, b, c, \ldots\}$ be a countable set of propositional variables. Then the set $\mathscr{F}$ of formulas is generated by

$$
\begin{aligned}
\mathscr{F}::= & \perp|1| \mathscr{A}\left|\mathscr{A}^{\perp}\right|[\mathscr{F} \& \mathscr{F}]|(\mathscr{F} \otimes \mathscr{F})| \\
& \forall \mathscr{A} \cdot \mathscr{F} \mid \exists \mathscr{A} \cdot \mathscr{F}
\end{aligned}
$$

\footnotetext{
${ }^{1}$ Note that second order propositional classical logic, i.e., "Quantified Boolean Formulas", is decidable (PSPACE-complete).
} 


$$
\begin{aligned}
& \text { id } \frac{\vdash \Gamma}{\vdash a^{\perp}, a} \quad \perp \frac{\vdash \Gamma}{\vdash \perp, \Gamma} \quad 1 \frac{}{\vdash 1} \quad \operatorname{exch} \frac{\vdash \Gamma, A, B, \Delta}{\vdash \Gamma, B, A, \Delta} \\
& \text { ४ } \frac{\vdash A, B, \Gamma}{\vdash[A \& B], \Gamma} \quad \otimes \frac{\vdash \Gamma, A \quad \vdash B, \Delta}{\vdash \Gamma,(A \otimes B), \Delta} \quad \exists \frac{\vdash A\langle a \backslash B\rangle, \Gamma}{\vdash \exists a \cdot A, \Gamma} \quad \forall \frac{\vdash A, \Gamma}{\vdash \forall a . A, \Gamma} \quad \begin{array}{l}
\text { in the } \forall \text {-rule, the } \\
\text { variable } a \text { must } \\
\text { not be free in } \Gamma
\end{array}
\end{aligned}
$$

Figure 1. Sequent calculus system for MLL2

Formulas are denoted by capital Latin letters $(A, B, C, \ldots)$. Linear negation $(-)^{\perp}$ is defined by the De Morgan laws:

$$
\begin{aligned}
\perp^{\perp} & =1 & 1^{\perp} & =\perp \\
a^{\perp} & =a^{\perp} & a^{\perp \perp} & =a \\
{[A 8 B]^{\perp} } & =\left(A^{\perp} \otimes B^{\perp}\right) & (A \otimes B)^{\perp} & =\left[A^{\perp} \ngtr B^{\perp}\right] \\
(\exists a . A)^{\perp} & =\forall a . A^{\perp} & (\forall a . A)^{\perp} & =\exists a . A^{\perp}
\end{aligned}
$$

Sequents are finite lists of formulas, separated by comma, and are denoted by capital Greek letters $(\Gamma, \Delta, \ldots)$. The notions of free and bound variable are defined in the usual way, and we can always rename bound variables.

In view of the later parts of the paper, and in order to avoid changing syntax all the time, we use the following syntactic conventions:

(i) We always put parentheses around binary connectives. For readability we use [...] for 8 and $(.$.$) for \otimes$.

(ii) We omit parentheses if they are superfluous under the assumption that $\varnothing$ and $\otimes$ associate to the left, e.g., $[A$ \& $B$ \& $C$ \& $D]$ abbreviates $[[[A$ \& $B] \& C]$ \& $D]$.

(iii) The scope of a quantifier ends at the earliest possible place (and not at the latest possible place as usual). This helps saving unnecessary parentheses. For example, in $[\forall a .(a \otimes b) \& \exists c . c \ngtr a]$, the scope of $\forall a$ is $(a \otimes b)$, and the scope of $\exists c$ is just $c$. In particular, the $a$ at the end is free.

The inference rules for MLL2 are shown in Figure 1 In the following, we will call this system MLL2Seq. As shown in [10], it has the cut elimination property:

$$
\begin{aligned}
& \text { 2.1 Theorem The cut rule } \\
& \qquad \operatorname{cut} \frac{\vdash \Gamma, A \quad \vdash A^{\perp}, \Delta}{\vdash \Gamma, \Delta}
\end{aligned}
$$

is admissible for system MLL2seq.

\section{MLL2 in the calculus of structures}

Let us now present a deductive system for MLL2 based on deep inference. We use the calculus of structures, in which the distinction between formulas and sequents disappears. This is the reason for the syntactic conventions introduced in the previous section

\footnotetext{
${ }^{2}$ In the literature on deep inference, e.g., [5] 11], the formula $\left(a \otimes\left[b \&\left(a^{\perp} \otimes c\right)\right]\right)$ would be written as $\left(a,\left[b,\left(a^{\perp}, c\right)\right]\right)$, while without
}

The inference rules now work directly (as rewriting rules) on the formulas. The system for MLL2 is shown in Figure 2 There, $S\{\}$ stands for an arbitrary (positive) formula context. We omit the braces if the structural parentheses fill the hole. E.g., $S[A \ngtr B]$ abbreviates $S\{[A \ngtr B]\}$. The system in Figure 2 is called MLL2 $\mathrm{DI} \downarrow$. We consider here only the so-called down fragment of the system, which corresponds to the cut-free system in the sequent calculus 3 Note that the $\forall$-rule of MLL2Seq is in MLL2DI decomposed into three pieces, namely, $\mathrm{e} \downarrow, \mathrm{u} \downarrow$, and $f \downarrow$. We also need an explicit rule for associativity which is in the sequent calculus "built in". The relation between the $\otimes$-rule and the rules Is and rs (called left switch and right switch) has already in detail been investigated by several authors [3] 9 11 [14]. A derivation $\mathscr{D}$ in the system MLL2 ${ }_{\mathrm{DI}} \downarrow$ is denoted by

$$
\begin{gathered}
A \\
\text { MLL2D } \downarrow \|_{B} \mathscr{D}
\end{gathered}
$$

and is simply a rewriting path from $A$ to $B$ using the inference rules in MLL2 ${ }_{\mathrm{D} \downarrow} \downarrow$. We say $A$ is the premise and $B$ the conclusion of $\mathscr{D}$. A proof in MLL2 $2 \mathrm{DI} \downarrow$ is a derivation whose premise is 1 . The following theorem ensures that MLL2DI is indeed a deductive system for MLL2.

3.1 Theorem For every proof of $\vdash A_{1}, \ldots, A_{n}$ in MLL2Seq, there is a proof of $\left[A_{1} 8 \ldots 8 A_{n}\right]$ in MLL2 ${ }_{\mathrm{DI} \downarrow}$, and vice versa.

As for MLL2seq, we also have for MLL2 $\mathrm{DI} \downarrow$ the cut elimination property, which can be stated as follows:

3.2 Theorem The cut rule (i.e., the "identity up rule")

$$
\text { i } \frac{S\left(A \otimes A^{\perp}\right)}{S\{\perp\}}
$$

is admissible for system MLL2DI .

our convention it would be written as $a \otimes\left(b \otimes\left(a^{\perp} \otimes c\right)\right)$. Our convention can therefore be seen as an attempt to please both communities. In particular, note that the motivation for the syntactic convention (iii) above is the collapse of the 8 on the formula level and the comma on the sequent level, e.g., $[\forall a .(a \otimes b) \ngtr \exists c . c \ngtr a]$ is the same as $[\forall a .(a, b), \exists c . c, a]$.

${ }^{3}$ The up fragment (which corresponds to the cut in the sequent calculus) is obtained by dualizing the rules in the down fragment, i.e., by negating

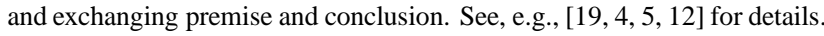




$$
\begin{aligned}
& \text { ai } \downarrow \frac{S\{1\}}{S\left[a^{\perp} 8 a\right]} \quad \quad \downarrow \frac{S\{A\}}{S[\perp 8 A]} \quad 1 \downarrow \frac{S\{A\}}{S(1 \otimes A)} \quad \text { e } \downarrow \frac{S\{1\}}{S\{\forall a .1\}} \\
& \alpha \downarrow \frac{S[[A \ngtr B] \ngtr C]}{S[A \ngtr[B \ngtr C]]} \quad \sigma \downarrow \frac{S[A \ngtr B]}{S[B \ngtr A]} \quad \text { Is } \frac{S([A \ngtr B] \otimes C)}{S[A \ngtr(B \otimes C)]} \quad \text { rs } \frac{S(A \otimes[B \ngtr C])}{S[(A \otimes B) \& C]} \quad \text { u } \frac{S\{\forall a .[A \ngtr B]\}}{S[\forall a \cdot A \ngtr \exists a \cdot B]} \\
& \mathrm{n} \downarrow \frac{S\{A\langle a \backslash B\rangle\}}{S\{\exists a . A\}} \quad \mathrm{f} \downarrow \frac{S\{\exists a \cdot A\}}{S\{A\}} \quad \text { In the } \mathrm{f} \downarrow \text {-rule, the variable } a \text { must not be free in } A \text {. }
\end{aligned}
$$

Figure 2. Deep inference system for MLL2

The following theorem states an important property of MLL2DI $\downarrow$ which has no counterpart in the sequent calculus.

$$
\begin{aligned}
& \text { 3.3 Theorem Every proof MLL2DI } \| \mathscr{D} \text { can be } \\
& \text { transformed into a proof } \\
& \{\text { ai } \downarrow, \perp \downarrow, 1 \downarrow, \mathrm{e} \downarrow\}
\end{aligned}
$$

for some $A$ and $B$.

This decomposition is obtained by permuting all instances of ai $\downarrow, \perp \downarrow, 1 \downarrow$, e $\downarrow$ up and permuting all instances of $\mathrm{n} \downarrow, \mathrm{f} \downarrow$ down. The attentive reader might wonder why there are two versions of the "switch" in MLL2DI $\downarrow$, the left switch Is, and the right switch rs. For completeness (Theorem 3.1, the Is-rule would be sufficient, but for obtaining the decomposition in Theorem 3.3 we need the rs-rule as well.

If a derivation $\mathscr{D}$ uses only the rules $\alpha \downarrow, \sigma \downarrow$, ls, rs, $\mathbf{u} \downarrow$, then premise and conclusion of $\mathscr{D}$ (and every formula in between the two) must contain the same atom occurrences. Hence, the atomic flow-graph [6] of the derivation $\mathscr{D}$ defines a bijection between the atom occurrences of premise and conclusion of $\mathscr{D}$. Here is an example of a derivation together with its flow-graph.

$$
\begin{aligned}
& \text { Is } \frac{\forall a . \forall b \cdot\left(\left[a^{\perp} \gamma a\right] \otimes\left[b^{\perp} \gamma b\right]\right)}{\forall a \cdot \forall b \cdot\left[a^{\perp} \gamma\left(a \otimes\left[b^{\perp} \ngtr b\right]\right)\right]}
\end{aligned}
$$

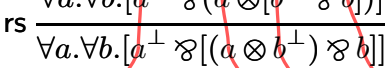

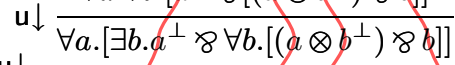

$$
\begin{aligned}
& u \downarrow \frac{u a \cdot\left[\exists b . a^{\perp} 8\left[\exists b .\left(a \otimes b^{\perp}\right) 8 \forall b . b\right)\right]}{\left[\forall a . \exists b . a^{\perp} 8 \exists a .\left[\exists b .\left(a \otimes b^{\perp}\right) 8 \forall b . b\right]\right]}
\end{aligned}
$$

To avoid crossings in the flow-graph, we left some applications of $\alpha \downarrow$ and $\sigma \downarrow$ implicit.

\section{Some observations on locality}

In the sequent calculus the $\forall$-rule has a non-local behavior, in the sense that for applying the rule we need some global knowledge about the context $\Gamma$, namely, that the variable $a$ does not appear freely in it. This is the reason for the existence of boxes in Girard's proof nets [10]. In the calculus of structures this "checking" whether a variable appears freely is done in the rule $f \downarrow$, which is as non-local as the $\forall$-rule. However, with deep inference, this rule can be made local, i.e., reduced to an atomic version (in the same sense as the identity axiom can be reduced to an atomic version). For this, we need an additional set of rules which is shown in Figure 3 (again, we show only the down fragment), and which is called $L f \downarrow$. Clearly, all rules are sound, i.e., proper implications of MLL2. Now we have the following:

\subsection{Theorem Everyderivation}

$$
\{\mathrm{n} \downarrow, \mathrm{f} \downarrow\} \|_{C}^{B} \mathscr{D} \text { can be transformed into } \quad\{\mathrm{n} \downarrow\} \cup \mathrm{Lf} \downarrow \|_{C}^{B} \mathscr{D}^{\prime},
$$

and vice versa.

There are seemingly two other sources of non-locality in MLL2DI $\downarrow$. The first is the renaming of bound variables $(\alpha-$ conversion) that might be necessary for applying $u \downarrow$ or $f \downarrow$ (or af $\downarrow$ ). However, if we see bound variables as pointers to the quantifier which binds them, as for example in DeBruijn presentation [8], then $\alpha$-conversion is no issue anymore 4 What remains is the substitution of an arbitrary formula for a variable in the instantiation of an existential quantifier (the $\mathrm{n} \downarrow$-rule), in which we have the unbounded number of occurrences of the quantified variable and the unbounded size of the formula to be substituted. It is a question of future research whether some sort of explicit substitution [1] can be coded directly into the inference rules in order to obtain a completely local system and by this closing the gap between the formal deductive system and the implementation.

\footnotetext{
${ }^{4}$ The only reason for not using DeBruijn presentation here is its unreadability for human beings.
} 


\begin{tabular}{|c|c|c|c|c|}
\hline$\times \frac{S\{\exists a \cdot \forall b \cdot A\}}{A(2)}$ & $\mathrm{y} \downarrow \frac{S\{\exists a \cdot \exists b \cdot A\}}{S(\neg)\urcorner}$ & $\mathrm{v} \downarrow \frac{S\{\exists a \cdot[A \ngtr B]\}}{S[\neg}$ & $\mathrm{w} \downarrow \frac{S\{\exists a \cdot(A \otimes B)\}}{}$ & \\
\hline$S\{\forall b . \exists a . A\}$ & $S\{\exists b . \exists a . A\}$ & $S[\exists a . A$ $\triangleleft a . B]$ & $S(\exists a . A \otimes \exists a . B)$ & \\
\hline $1 \mathrm{f}\rfloor \underline{S\{\exists a .1\}}$ & $S\{\exists a . \perp\}$ & $S\{\exists a . b\}$ & $S\left\{\exists a . b^{\perp}\right\}$ & in af $\downarrow$ and âf $\downarrow$, \\
\hline$S\{1\}$ & $\perp \downarrow \overline{S\{\perp\}}$ & $S\{b\}$ & $S\left\{b^{\perp}\right\}$ & different from $b$ \\
\hline
\end{tabular}

Figure 3. Towards a local system for MLL2

\section{Proof nets for MLL2}

For defining proof nets for MLL2, we follow the ideas presented in [21 17] where the axiom linking of multiplicative proof nets has been replaced by a linking formula to accommodate the units 1 and $\perp$. In such a linking formula, the ordinary axiom links are replaced by $\otimes$-nodes, which are then connected by $8 \mathrm{~s}$. A unit can then be attached to a sublinking by another $\otimes$, and so on. Here we extend the syntax for the linking formula by an additional construct to accommodate the quantifiers. Now, the set $\mathscr{L}$ of linking formulas is generated by the grammar

$$
\mathscr{L}::=\perp\left|\left(\mathscr{A} \otimes \mathscr{A}^{\perp}\right)\right|(1 \otimes \mathscr{L})|[\mathscr{L} \otimes \mathscr{L}]| \exists \mathscr{A} \cdot \mathscr{L}
$$

In [21, 17] a proof net consists of the sequent forest and the linking formula. The presence of the quantifiers, in particular, the presence of instantiation and substitution, makes it necessary to expand the structure of the sequent in the proof net. The set $\mathscr{E}$ of expanded formula 5 is generated by

$$
\begin{aligned}
\mathscr{E}::= & \perp|1| \mathscr{A}\left|\mathscr{A}^{\perp}\right|[\mathscr{E} \otimes \mathscr{E}]|(\mathscr{E} \otimes \mathscr{E})| \\
& \forall \mathscr{A} \cdot \mathscr{E}|\exists \mathscr{A} \cdot \mathscr{E}| \Xi \mathscr{A} \cdot \mathscr{E} \mid \exists \mathscr{A} . \mathscr{E}
\end{aligned}
$$

There are only two additional syntactic primitives: the $\Xi$, called virtual existential quantifier, and the $\exists$, called bold existential quantifier. An expanded sequent is a finite list of expanded formulas, separated by comma. We denote expanded sequents by capital Greek letters $(\Gamma, \Delta, \ldots)$. For disambiguation, the formulas/sequents introduced in Section 2 (i.e., those without $\exists$ and $\exists$ ) will also be called simple formulas/sequents.

In the following we will identify formulas with their syntax trees, where the leaves are decorated by elements of $\mathscr{A} \cup \mathscr{A}^{\perp} \cup\{1, \perp\}$. We can think of the inner nodes as decorated either with the connectives/quantifiers $\otimes, \diamond, \forall a, \exists a$, $\exists a, \exists a$, or with the whole subformula rooted at that node. For this reason we will use capital Latin letters $(A, B, C$, ...) to denote nodes in a formula tree. We write $A \leqslant B$ if $A$ is a (not necessarily proper) ancestor of $B$, i.e., $B$ is a subformula occurrence in $A$. We write ${ }^{\circ}$ (resp. $A$ ) for denoting the set of leaves of a sequent $\Gamma$ (resp. formula $A$ ).

\footnotetext{
${ }^{5}$ There is some similarity to Miller's expansion trees 18 . The idea is to code a formula and its "expansion" together in the same syntactic object. But our case is simpler than in 18 because we do not have to deal with duplication.
}

5.1 Definition Let $\Gamma$ be an expanded sequent. A stretching $\sigma$ for $\Gamma$ consists of two binary relations $\stackrel{\sigma}{\sim}$ and $\stackrel{\sigma}{\curvearrowleft}$ on the set of nodes of $\Gamma$ (i.e., the set of its subformula occurrences) such that $\stackrel{\sigma}{\sim}$ and $\stackrel{\sigma}{\sim}$ are disjoint, and whenever $A \stackrel{\sigma}{\sim} B$ or $A \stackrel{\sigma}{\sigma} B$ then $A=\exists a . A^{\prime}$ with $A^{\prime} \leqslant B$ in $\Gamma$.

In other words, a stretching consists of edges connecting $\exists$-nodes with some of its subformulas, and these edges can be directed towards the $\exists$-node or away from it. When writing an expanded sequent $\Gamma$ with a stretching $\sigma$, shortly denoted by $\Gamma \triangleleft \sigma$, we will draw these edges either inside $\Gamma$ when it is written as a tree, or below $\Gamma$ when it is written as string. Examples are shown in Figures 46 and 7 below.

Now we are ready to see the definition of a pre-proof graph. The "pre-" means that we do not yet know whether it really comes from an actual proof

5.2 Definition A pre-proof graph is a quadruple, denoted by $P \stackrel{\nu}{\triangleright} \Gamma \triangleleft \sigma$, where $P$ a linking formula, $\Gamma$ is an expanded sequent, $\sigma$ is a stretching for $\Gamma$, and $\nu$ is a bijection ${ }^{\circ} \stackrel{\nu}{\rightarrow}$ 四 such that only dual atoms/units are paired up. If $\Gamma$ is simple, we say that the pre-proof graph is simple. In this case $\sigma$ is empty, and we can simply write $P \stackrel{\nu}{\triangleright} \Gamma$.

For $B \in$ we write $B^{\nu}$ for its image under $\nu$ in $P$. When we draw a pre-proof graph $P \stackrel{\nu}{\triangleright} \Gamma \triangleleft \sigma$, then (following the tradition) the roots of the formula trees in $\Gamma$ will be drawn at the bottom below the leaves, and the root of $P$ will be drawn above the leaves. The leaves of $P$ and $\Gamma$ are connected by edges according to $\nu$. In order to save space we can also write $P$ and $\Gamma$ as strings above each other. Figure 4 shows an example written in both ways.

Let us now turn our attention towards correctness. For this we concentrate first on simple pre-proof graphs and begin with the standard notion of multiplicative correctness:

5.3 Definition A DR-switching $s$ of a simple pre-proof graph $P \stackrel{\nu}{\triangleright} \Gamma$ is the graph that is obtained from $P \stackrel{\nu}{\triangleright} \Gamma$ by removing for each 8 -node one of the two edges connecting it to its children. A simple pre-proof graph $P \stackrel{\nu}{\triangleright} \Gamma$ is multiplicatively correct if all its DR-switchings are acyclic

\footnotetext{
${ }^{6}$ The concept of a "not yet proof" is in the literature (e.g., [7) also called "proof structure".
} 

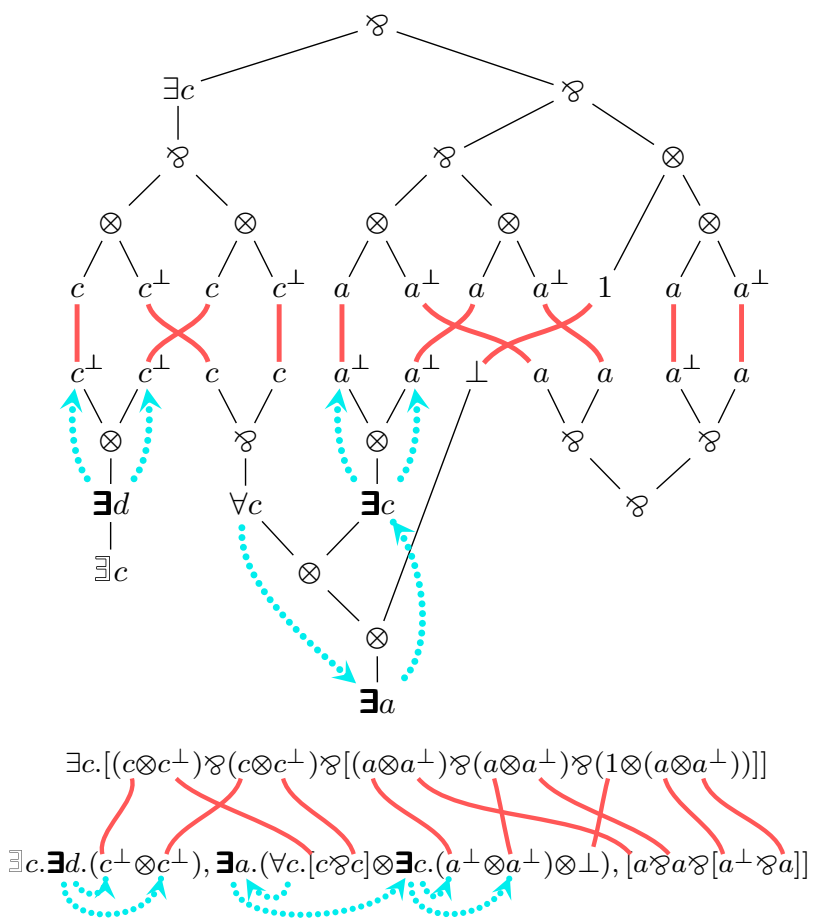

Figure 4. Two ways of writing a proof graph

and connected [7]. For a pre-proof graph $P \stackrel{\nu}{\triangleright} \Gamma \triangleleft \sigma$ we define multiplicative correctness accordingly, but we ignore the edges of the stretching when checking acyclicity and connectedness.

Note that for multiplicative correctness the quantifiers are treated as unary connectives and are therefore completely irrelevant. Clearly, the example in Figure 4 is multiplicatively correct. However, for involving the quantifiers into a correctness criterion, we need some more conditions.

Let $s$ be a DR-switching for $P \stackrel{\nu}{\triangleright} \Gamma$, and let $A$ and $B$ be two nodes in $\Gamma$. We write $A \Omega S B$ if there is a path in $s$ from $A$ to $B$, starting from $A$ by going down to its parent and coming into $B$ from below. Similarly, one can define the notations $A$ (s) $B$ and $A$ s $B$ and $A$ s? $B$.

Let $A$ and $B$ be nodes in $\Gamma$ with $A \leqslant B$. The quantifier depth of $B$ in $A$, denoted by $\nabla_{A} B$, is the number of quantifier nodes on the path from $A$ to $B$ (including $A$ if it happens to be an $\forall$ or an $\exists$ ). Similarly we define $\nabla_{\Gamma} B$. For quantifier nodes $A^{\prime}$ in $P$ and $A$ in $\Gamma$, we say $A$ and $A^{\prime}$ are partners, denoted by $A^{\prime} \stackrel{P}{\longrightarrow} A$, if there is a leaf $B \in \mathbb{Q}$ with $A \leqslant B$ in $\Gamma$, and $A^{\prime} \leqslant B^{\nu}$ in $P$, and $\nabla_{A} B=\nabla_{A^{\prime}} B^{\nu}$.

5.4 Definition We say a simple pre-proof graph $P \stackrel{\nu}{\triangleright} \Gamma$ is well-nested if the following five conditions are satisfied:

1. For every $B \in$ Q $\Gamma$, we have $\nabla_{\Gamma} B=\nabla_{P} B^{\nu}$.

2. If $A^{\prime} \stackrel{P}{\longrightarrow} A$, then $A^{\prime}$ and $A$ quantify the same variable.

3. For every quantifier node $A$ in $\Gamma$ there is exactly one $\exists$-node $A^{\prime}$ in $P$ with $A^{\prime} \stackrel{P}{\rightleftarrows} A$.
4. For every $\exists$-node $A^{\prime}$ in $P$ there is exactly one $\forall$-node $A$ in $\Gamma$ with $A^{\prime} \stackrel{P}{\longrightarrow} A$.

5. If $A^{\prime} \stackrel{P_{2}}{\longmapsto} A_{1}$ and $A^{\prime} \stackrel{P}{\longmapsto} A_{2}$, then there is no DRswitching $s$ with $A_{1} \Omega A_{2}$.

Every quantifier node in $P$ must be an $\exists$, and every quantifier node in $\Gamma$ has exactly one of them as partner. On the other hand, an $\exists$ in $P$ can have many partners in $\Gamma$, but exactly one of them has to be an $\forall$. Following Girard [10], we can call an $\exists$ in $P$ together with its partners in $\Gamma$ the doors of an $\forall$-box and the sub-graph induced by the nodes that have such a door as ancestor is called the $\forall$-box associated to the unique $\forall$-door. Even if the boxes are not really present, we can use the terminology to relate our work to Girard's. Furthermore, all the properties of these boxes that are postulated in [10], e.g., that every box is correct in itself, follow from the global multiplicative correctness and the five conditions above. In order to help the reader to understand these five conditions, we show in Figure 5 six simple pre-proof graphs, where the first fails Condition 1 the second one fails Condition 2 and so on; only the sixth one is well-nested.

5.5 Definition A simple pre-proof graph $P \stackrel{\nu}{\triangleright} \Gamma$ is correct if it is well-nested and multiplicatively correct. In this case we will also speak of a simple proof graph.

Let us now turn our attention towards substitution, which is the raison d'être for the expansion with $\exists$ and $\Xi$.

5.6 Definition For an expanded formula $E$ and a stretching $\sigma$, we define the ceiling and the floor 7 , denoted by $\lceil E \triangleleft \sigma\rceil$ and $\lfloor E \triangleleft \sigma\rfloor$, respectively, to be simple formulas, which are inductively defined as follows:

$$
\begin{aligned}
& \lceil 1 \triangleleft \emptyset\rceil=1 \quad\lceil A \ngtr B \bullet \sigma\rceil=\left\lceil A \bullet \sigma^{\prime}\right\rceil 8\left\lceil B \triangleleft \sigma^{\prime \prime}\right\rceil \\
& \lceil\perp \triangleleft \emptyset\rceil=\perp \quad\lceil A \otimes B \triangleleft \sigma\rceil=\left\lceil A \triangleleft \sigma^{\prime}\right\rceil \otimes\left\lceil B \triangleleft \sigma^{\prime \prime}\right\rceil \\
& \lceil a \triangleleft \emptyset\rceil=a \quad\lceil\forall a . A \hookrightarrow \sigma\rceil=\forall a .\lceil A \hookrightarrow \sigma\rceil \\
& \left\lceil a^{\perp} \triangleleft \emptyset\right\rceil=a^{\perp} \quad\lceil\exists a . A \triangleleft \sigma\rceil=\exists a .\lceil A \triangleleft \sigma\rceil \\
& \lceil\Xi a . A \hookrightarrow \sigma\rceil=\exists a .\lceil A \bullet \sigma\rceil \\
& \lceil\exists a . A \bullet \sigma\rceil=\left\lceil A \triangleleft \sigma^{\prime}\right\rceil \\
& \lfloor 1 \bullet \emptyset\rfloor=1 \quad\lfloor A \ngtr B \bullet \sigma\rfloor=\left\lfloor A \bullet \sigma^{\prime}\right\rfloor \ngtr\left\lfloor B \bullet \sigma^{\prime \prime}\right\rfloor \\
& \lfloor\perp \bullet \emptyset\rfloor=\perp \quad\lfloor A \otimes B \triangleleft \sigma\rfloor=\left\lfloor A \hookrightarrow \sigma^{\prime}\right\rfloor \otimes\left\lfloor B \triangleleft \sigma^{\prime \prime}\right\rfloor \\
& \lfloor a \triangleleft \emptyset\rfloor=a \quad\lfloor\forall a . A \bullet \sigma\rfloor=\forall a .\lfloor A \bullet \sigma\rfloor \\
& \left\lfloor a^{\perp} \bullet \emptyset\right\rfloor=a^{\perp} \quad\lfloor\exists a . A \hookrightarrow \sigma\rfloor=\exists a .\lfloor A \hookrightarrow \sigma\rfloor \\
& \lfloor\Xi a . A \bullet \sigma\rfloor=\lfloor A \bullet \sigma\rfloor \\
& \lfloor\exists a . A \bullet \sigma\rfloor=\exists a .\lfloor\tilde{A} \bullet \tilde{\sigma}\rfloor
\end{aligned}
$$

where $\sigma^{\prime}$ is always the restriction of $\sigma$ to $A$, and $\sigma^{\prime \prime}$ is always the restriction of $\sigma$ to $B$. The expanded formula $\tilde{A}$ in the last line is obtained from $A$ as follows: For every node $B$ with $A \leqslant B$ and $\exists a . A \stackrel{\sigma}{\sim} B$ remove the whole subtree $B$ and replace it by $a$, and for every $B$ with $\exists a . A \stackrel{\sigma}{\llcorner} B$

\footnotetext{
${ }^{7}$ These correspond to Miller's functions Deep and Shallow 18 .
} 
(1)

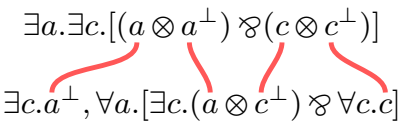

(4)

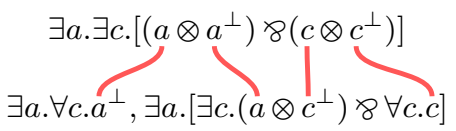

$(2)$

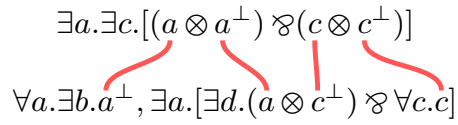

(5)

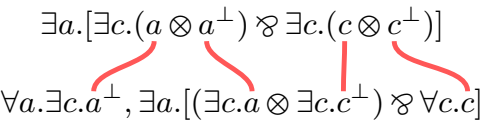

(3)

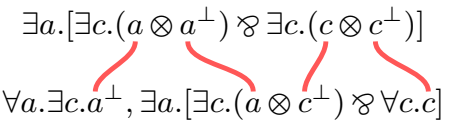

(6)

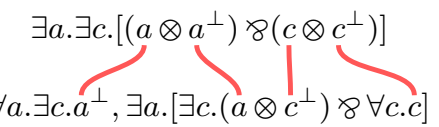

Figure 5. Examples (1)-(5) are not well-nested, only (6) is well-nested

replace $B$ by $a^{\perp}$. The stretching $\tilde{\sigma}$ is the restriction of $\sigma$ to $\tilde{A}$. For an expanded sequent $\Gamma$, we proceed analogously.

Note that ceiling and floor of an expanded sequent $\Gamma$ differ from $\Gamma$ only on $\exists$ and $\Xi$. In the ceiling, the $\exists$ is treated as ordinary $\exists$, and the $\exists$ is completely ignored. In the floor, the פ is ignored, and the $\exists$ uses the information of the stretching to "undo the substitution". To provide this information on the location is the only purpose of the stretching. However, to ensure that we really only "undo the substitution" instead of doing something weird, we need some further constraints, which are given by Definition 5.7 below.

Let $\Gamma \triangleleft \sigma$ be given, and let $A$ and $B$ be nodes in $\Gamma$ with $A$ being a quantifier node and $A \preccurlyeq B$. Then we write $A \curvearrowleft B$ if $A$ is a $\exists$-node and there is a stretching edge between $A$ and $B$, or $A$ is an ordinary quantifier node and $B$ is the variable (or its negation) that is bound in $A$.

5.7 Definition A pair $\Gamma \triangleleft \sigma$ is appropriate, if the following three conditions hold:

1. If $A \stackrel{\sigma}{\sim} B_{1}$ and $A \stackrel{\sigma}{\triangleleft} B_{2}$, then $\left\lfloor B_{1} \triangleleft \sigma_{1}\right\rfloor=\left\lfloor B_{2} \triangleleft \sigma_{2}\right\rfloor$, if $A \stackrel{\sigma}{\sim} B_{1}$ and $A \stackrel{\sigma}{\sim} B_{2}$, then $\left\lfloor B_{1} \triangleleft \sigma_{1}\right\rfloor=\left\lfloor B_{2} \triangleleft \sigma_{2}\right\rfloor$, if $A \stackrel{\sigma}{\triangle} B_{1}$ and $A \stackrel{\sigma}{\sim} B_{2}$, then $\left\lfloor B_{1} \triangleleft \sigma_{1}\right\rfloor=\left\lfloor B_{2} \triangleleft \sigma_{2}\right\rfloor^{\perp}$, (where $\sigma_{1}$ and $\sigma_{2}$ are the restrictions of $\sigma$ to $B_{1}$ and $B_{2}$, respectively).

2. If $A_{1} \bowtie B_{1}$ and $A_{2} \curvearrowleft B_{2}$ and $A_{1} \preccurlyeq A_{2}$ and $B_{1} \preccurlyeq B_{2}$, then $B_{1} \preccurlyeq A_{2}$.

3. For all $\Xi a$. $A$, the variable $a$ must not occur free in the formula $\left\lfloor A \triangleleft \sigma^{\prime}\right\rfloor$ (where $\sigma^{\prime}$ is the restriction of $\sigma$ to $A$ ).

The first condition above says that in a substitution a variable is instantiated everywhere by the same formula $B$. The second condition ensures that there is no variable capturing in such a substitution step. The third condition is exactly the side condition of the rule $f \downarrow$ in Figure 2 For better explaining the three conditions above, we show in Figure 6 three examples of pairs $\Gamma \triangleleft \sigma$ that are not appropriate: the first fails Condition 1 the second fails Condition 2 and the third fails Condition 3 In Figure 7 all three examples are appropriate. The example in Figure (4) is also appropriate.

In [10], the first two conditions if Definition 5.7 appear only implicitly without being mentioned in the treatment of the $\exists$-rule. However, for capturing the essence of a proof
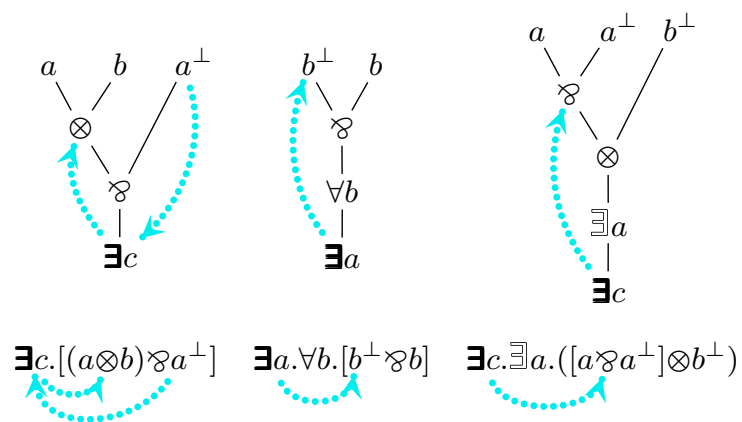

Figure 6. Examples of expanded sequents with stretchings that are not appropriate
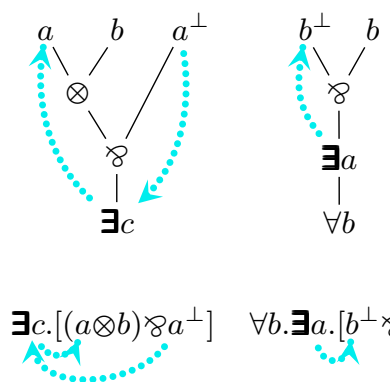

$\forall b . \exists a \cdot\left[b^{\perp} \varnothing b\right]$

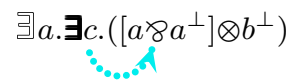

Figure 7. Appropriate examples of expanded sequents with stretchings

independently of a deductive system, we have to make everything explicit.

5.8 Definition We say that a pre-proof graph $P \stackrel{\nu}{\triangleright} \Gamma \triangleleft \sigma$ is correct if the simple pre-proof graph $P \stackrel{\nu}{\triangleright}\lceil\Gamma \triangleleft \sigma\rceil$ is correct and the pair $\Gamma \triangleleft s$ is appropriate. In this case we say that $P \stackrel{\nu}{\triangleright} \Gamma \triangleleft \sigma$ is a proof graph and $\lfloor\Gamma \triangleleft \sigma\rfloor$ is its conclusion.

The example in Figure 4 is correct. There $\lceil\Gamma \triangleleft \sigma\rceil$ is

$\vdash \exists c .\left(c^{\perp} \otimes c^{\perp}\right),\left(\forall c .[c \ngtr c] \otimes\left(a^{\perp} \otimes a^{\perp}\right) \otimes \perp\right),\left[a \ngtr a \ngtr\left[a^{\perp} \ngtr a\right]\right]$ and the conclusion $\lfloor\Gamma \triangleleft \sigma\rfloor$ is

$$
\vdash \exists d .(d \otimes d), \exists a \cdot\left(a^{\perp} \otimes a \otimes \perp\right),\left[a>a \ngtr\left[a^{\perp} 8 a\right]\right] \quad .
$$

Due to the presence of the multiplicative units, we need to enforce an equivalence relation on proof graphs. 
5.9 Definition Let $\sim$ be the smallest equivalence relation on the set of proof graphs satisfying

$$
\begin{aligned}
& P[Q \ngtr R] \stackrel{\nu}{\llcorner} \Gamma \bullet \sigma \sim P[R \ngtr Q] \stackrel{\nu}{\triangleright} \Gamma \bullet \sigma \\
& P[[Q \ngtr R] \ngtr S] \stackrel{\nu}{\triangleright} \Gamma \bullet \sigma \sim P[Q \ngtr[R \ngtr S]] \stackrel{\nu}{\triangleright} \Gamma \bullet \sigma \\
& P(1 \otimes(1 \otimes Q)) \stackrel{\nu}{\triangleright} \Gamma \bullet \sigma \sim P(1 \otimes(1 \otimes Q)) \stackrel{\nu^{\prime}}{\triangleright} \Gamma \bullet \sigma \\
& P(1 \otimes[Q \ngtr R]) \stackrel{\nu}{\triangleright} \Gamma \triangleleft \sigma \sim P[(1 \otimes Q) \& R] \stackrel{\nu}{\triangleright} \Gamma \triangleleft \sigma \\
& P(1 \otimes \exists a . Q) \stackrel{\nu}{\triangleright} \Gamma\{\perp\} \bullet \sigma \sim P\{\exists a .(1 \otimes Q)\} \stackrel{\nu}{\triangleright} \Gamma\{\exists a . \perp\} \bullet \sigma
\end{aligned}
$$

where in the third line $\nu^{\prime}$ is obtained from $\nu$ by exchanging the preimages of the two 1s. In all other equations the bijection $\nu$ does not change. In the last line $\nu$ must match the 1 and $\perp$. A proof net is an equivalence class of $\sim$.

The first two equations in Definition 5.9 are simply associativity and commutativity of 8 inside the linking. The third is a version of associativity of $\otimes$. The fourth equation could destroy multiplicative correctness, but since we defined $\sim$ only on proof graphs we do not need to worry about that 8 The last equation says that a $\perp$ can freely tunnel through the borders of a box. Let us emphasize that this quotienting via an equivalence is due to the multiplicative units. If one wishes to use a system without units, one could completely dispose the equivalence by using $n$-ary $8 \mathrm{~s}$ in the linking.

\section{Sequentialisation}

In this section we will discuss how we can translate proofs in the sequent calculus and the calculus of structures into proof nets, and how we can read back a deductive proof from a proof net.

For traditional reasons let us begin with the sequent calculus. The translation from MLL2seq proofs into proof graphs is done inductively on the structure of the sequent proof as shown in Figure 8 For the rules id and 1, this is trivial ( $\nu_{0}$ and $\nu_{1}$ are uniquely determined and the stretching is empty). In the rule $\perp$, the $\nu_{\perp}$ is obtained from $\nu$ by adding an edge between the new 1 and $\perp$. The exch and 8 rules are also rather trivial ( $P, \nu$, and $\sigma$ remain unchanged). For the $\otimes$ rule, the two linkings are connected by a new 8 node, and the two principal formulas are connected by a $\otimes$ in the sequent forest. The two interesting rules are the ones for $\forall$ and $\exists$. In the $\forall$-rule, to every root node of the proof graph for the premise a quantifier node is attached. This is what ensures the well-nestedness condition. It can be compared to Girard's putting a box around a proof net. The purpose of the $\exists$ can be interpreted as simulating the border of the box. The $\exists$-rule is the only one where the stretching $\sigma$ is changed. As shown in Figure 1 in the conclusion of that rule, the subformula $B$ of $A$ is replaced by the quantified variable $a$. When translating this rule into proof graphs,

\footnotetext{
${ }^{8} \operatorname{In}[21 \quad 17]$ the relation $\sim$ is defined on pre-proof graphs, and therefore a side condition had to be given to that equation (see also 13 ).
}

we do not do this replacement. We keep the $B$, but to every place where it has to be substituted we add a stretching edge from the new $\exists a$. Similarly, whenever a $B^{\perp}$ should be replaced by $a^{\perp}$, we add an edge from this position to the $\exists a$. The new stretching is $\sigma^{\prime}$. Finally, the cut is treated similar to the $\otimes$-rule. It will be discussed in the next section.

We will call a pre-proof graph $S C$-sequentializable if it can be obtained from a sequent proof as described above. Note that if a pre-proof graph $P \stackrel{\nu}{\triangleright} \Gamma \triangleleft \sigma$ is obtained this way then the simple sequent $\lfloor\Gamma \triangleleft \sigma\rfloor$ is exactly the conclusion of the sequent proof we started from. We have immediately

6.1 Theorem Every SC-sequentializable pre-proof graph is a proof graph.

For the other direction, i.e, for going from proof graphs to MLL2seq proofs we need to consider two linking formulas $P_{1}$ and $P_{2}$ to be equivalent modulo associativity and commutativity of $\ngtr$. We write this as $P_{1} \stackrel{\gtrless}{\sim} P_{2}$. Then, we have to remove all $\exists$-nodes from $\Gamma$ in order to get a sequentialization theorem because the translation shown in Figure 8 never introduces an $\exists$-node in $\Gamma$. For this we replace in $\Gamma$ every $\exists a . A$ with $\Xi a . \exists a$. $A$ and by add a stretching edge between the new $\exists a$ and every $a$ and $a^{\perp}$ that was previously bound by $\exists a$ (i.e, is free in $A$ ). Let us write $\widehat{\Gamma \hookrightarrow \sigma}$ for the result of this modification applied to $\Gamma \triangleleft \sigma$.

6.2 Theorem If $P \stackrel{\nu}{\triangleright} \Gamma \triangleleft \sigma$ is correct, then there is a $P^{\prime} \stackrel{\gtrless}{\sim} P$, such that $P^{\prime} \stackrel{\nu}{\triangleright} \widehat{\Gamma \leftarrow \sigma}$ is $S C$-sequentializable.

The proof works in the usual way by induction on the size of $P \stackrel{\nu}{\triangleright} \Gamma \triangleleft \sigma$. It is a combination of the sequentialization proofs in [17] and [10], and it makes crucial use of the "splitting tensor lemma" which in our case also needs the well-nestedness condition.

Let us now discuss the translation between proof nets and derivations in the calculus of structures. This can be done in a more modular way than for the sequent calculus. Let us start with some properties of linking formulas:

6.3 Proposition An MLL2 formula $P$ is a linking formula if and only if there is a derivation

$$
\{\text { ai } \downarrow, \perp \downarrow, 1 \downarrow, \mathrm{e} \downarrow\} \|_{P^{\perp}}^{1} \mathscr{D} .
$$

6.4 Lemma Let $P_{1}$ and $P_{2}$ be two linkings. Then there is a derivation

$$
\begin{gathered}
P_{1} \\
\{\alpha \downarrow, \sigma \downarrow, \mathrm{rs}\} \|_{P_{2}} \mathscr{D}
\end{gathered}
$$

if and only if the simple pre-proof graph $P_{2} \triangleright P_{1}^{\perp}$ is correct.

If $P_{1}$ and $P_{2}$ have this property, we say that $P_{1}$ is weaker than $P_{2}$, and denote it as $P_{1} \lesssim P_{2}$. We can now characterize simple proof graphs in terms of deep inference as follows: 


$$
\begin{aligned}
& \text { id } \overline{a \otimes a^{\perp} \stackrel{\nu_{0}}{\triangleright} a^{\perp}, a \triangleleft \emptyset} \\
& \operatorname{exch} \frac{P \stackrel{\nu}{\triangleright} \Gamma, A, B, \Delta \triangleleft \sigma}{P \stackrel{\nu}{\triangleright} \Gamma, B, A, \Delta \triangleleft \sigma} \\
& \forall \frac{P \stackrel{\nu}{\triangleright} A, B_{1}, \ldots, B_{n} \triangleleft \sigma}{\exists a . P \stackrel{\nu}{\triangleright} \forall a . A, \Xi a \cdot B_{1}, \ldots, \text { 引 } a \cdot B_{n} \triangleleft \sigma}
\end{aligned}
$$$$
\perp \frac{P \stackrel{\nu}{\triangleright} \Gamma \triangleleft \sigma}{(1 \otimes P)^{\nu} \stackrel{\nu}{\triangleright} \Gamma, \perp \triangleleft \sigma}
$$$$
1 \frac{\nu_{1}}{\perp \triangleright 1 \triangleleft \emptyset}
$$$$
8 \frac{P \stackrel{\nu}{\triangleright} A, B, \Gamma \triangleleft \sigma}{P_{\triangleright}^{\nu}[A \ngtr B], \Gamma \triangleleft \sigma}
$$$$
\otimes \frac{P \stackrel{\nu}{\triangleright} \Gamma, A \triangleleft \sigma \quad Q \stackrel{f}{\triangleright} B, \Delta \triangleleft \tau}{[P \ngtr Q] \stackrel{e \cup f}{\triangleright} \Gamma,(A \otimes B), \Delta \triangleleft \sigma \cup \tau}
$$$$
\exists \frac{P \stackrel{\nu}{\triangleright} \Gamma, A\langle a \backslash B\rangle \triangleleft \sigma}{P^{\nu} \Gamma, \exists a \cdot A\langle a \backslash B\rangle \triangleleft \sigma^{\prime}}
$$$$
\operatorname{cut} \frac{P \stackrel{\nu}{\triangleright} \Gamma, A \triangleleft \sigma \quad Q \stackrel{f}{\triangleright} A^{\perp}, \Delta \triangleleft \tau}{[P \ngtr Q] \stackrel{e \cup f}{\triangleright} \Gamma,\left(A \oplus A^{\perp}\right), \Delta \triangleleft \sigma \cup \tau}
$$

\section{Figure 8. Translating sequent calculus proofs into proof nets}

6.5 Proposition A simple pre-proof graph $P \stackrel{\nu}{\triangleright} \Gamma$ is correct if and only if there is a linking $P^{\prime}$ with $P^{\prime} \lesssim P$ and a derivation

$$
\{\alpha \downarrow, \sigma \downarrow, \mathrm{ls}, \mathrm{rs}, \mathrm{u} \downarrow\} \|_{\Gamma} P^{\prime \perp},
$$

such that $\nu$ coincides with the bijection induced by the flow graph of $\mathscr{D}$.

As an example for this proposition, consider the derivation in (4) which corresponds to example (6) in Figure 5

Finally, we characterize appropriate pairs $\Gamma \triangleleft \sigma$ in terms of deep inference.

\subsection{Proposition For every derivation}

$$
\begin{gathered}
D \\
\{\mathrm{n} \downarrow, \mathrm{f} \downarrow\} \\
\|_{C} \mathscr{D}
\end{gathered}
$$

there is an appropriate pair $\Gamma \triangleleft \sigma$ with

$$
D=\lceil\Gamma \triangleleft \sigma\rceil \text { and } C=\lfloor\Gamma \triangleleft \sigma\rfloor .
$$

Conversely, if $\Gamma \triangleleft \sigma$ is appropriate, then there is a derivation (7) with (8).

We can explain the idea of this proposition by considering again the examples in Figures 6 and 7 To the nonappropriate examples in Figure 6 would correspond the following incorrect derivations:

$$
\mathrm{n} \downarrow \frac{\left[(a \otimes b) \ngtr a^{\perp}\right]}{\exists c .\left[c \ngtr c^{\perp}\right]} \quad \mathrm{n} \downarrow \frac{\forall b .\left[b^{\perp} \ngtr b\right]}{\exists a . \forall b .[a \ngtr b]} \quad \mathrm{f} \downarrow \frac{\exists a \cdot\left(\left[a \ngtr a^{\perp}\right] \otimes b\right)}{\mathrm{n} \downarrow}
$$

And to the appropriate examples in Figure 7 correspond the following correct derivations:

$\mathrm{n} \downarrow \frac{\left[(a \otimes b) 8 a^{\perp}\right]}{\exists c .\left[(c \otimes b) 8 c^{\perp}\right]} \quad \mathrm{n} \downarrow \frac{\forall b \cdot\left[b^{\perp} 8 b\right]}{\forall b . \exists a \cdot[a \ngtr b]} \quad \mathrm{n} \downarrow \frac{\exists a \cdot\left(\left[a \ngtr a^{\perp}\right] \otimes b\right)}{\mathrm{f} \downarrow}$
We can now easily translate a MLL2 $\mathrm{DI} \downarrow$ proof into a preproof graph by first decomposing it into the shape 3 via Theorem 3.3 and then applying Propositions 6.36 .5 and 6.6 We get a pre-proof graph $P \stackrel{\nu}{\triangleright} \Gamma \triangleleft \sigma$ with $P^{\perp}=A$ and $\lceil\Gamma \triangleleft \sigma\rceil=B$ and $\lfloor\Gamma \triangleleft \sigma\rfloor=C$. Let us call a pre-proof graph DI-sequentializable if is obtained in this way from a MLL2DI $\downarrow$ proof. We immediately have

6.7 Theorem Every DI-sequentializable pre-proof graph is a proof graph.

By the method presented in [20], it is also possible to translate a MLL2 2 I $\downarrow$ directly into a proof graph without prior decomposition. However, the decomposition is the key for the translation from proof graphs into MLL2 2 I $\downarrow$ proofs (i.e., "sequentialization" into the calculus of structures). Propositions 6.36 .5 and 6.6 give us the following:

6.8 Theorem If $P \stackrel{\nu}{\triangleright} \Gamma \triangleleft \sigma$ is correct, then there is a $P^{\prime} \lesssim P$, such that $P^{\prime} \stackrel{\nu}{\triangleright} \Gamma \triangleleft \sigma$ is DI-sequentializable.

There is an important difference between the two sequentializations. While for the sequent calculus we have a monolithic procedure reducing the proof graph node by node, we have for the calculus of structures a modular procedure that treats the different parts of the proof graph (which correspond to the three different aspects of the logic) separately. The core is Proposition 6.5 which deals with the purely multiplicative part. Then comes Proposition 6.6 which only deals with instantiation and substitution, i.e, the second-order aspect. Finally, Proposition 6.3 takes care of the linking, whose task is to describe the role of the units in the proof. Therefore the equivalence in 5.9 which is due to the mobility if the $\perp$, only deals with the linkings. This modularity in the sequentialization is possible because of the decomposition in Theorem 3.3 which can be seen as a version of Herbrand's theorem.

Note that for both sequentializations only part of the equivalence 5.9 is needed. However, cut elimination in the next section relies of the full power of this equivalence. 


\section{Cut elimination}

A cut in a proof graph $P \stackrel{\nu}{\triangleright} \Gamma \triangleleft \sigma$ is a special binary connective $\Phi$, such that whenever we have $A \oplus B$ in $\Gamma$, then we must have $\lfloor A \hookrightarrow \sigma\rfloor=\lfloor B \triangleleft \sigma\rfloor^{\perp}$. Morally, a $(1)$ may occur only at the root of a formula in $\Gamma$. However, due to well-nestedness we must allow cuts to have 引-nodes as ancestors. Then the $\Phi$ is treated in the correctness criterion in exactly the same way as the $\otimes$, and sequentialization does also hold for proof graphs with cut.

The cut reduction relation $\sim$ is defined on proof graphs as shown in Figure 9 For cuts not involving quantifiers we proceed as in [17]: If we have an atomic cut $a^{\perp}(1 a$, then we must have in $P$ two "axiom links" $\left(a^{\perp} \otimes a\right)$, which are by the leaf mapping $\nu$ attached to the two atoms in the cut. Via the equivalence in 5.9 we can bring the two pairs next to each other such that $P$ has $\left[\left(a \otimes a^{\perp}\right) \&\left(a \otimes a^{\perp}\right)\right]$ as subformula. We can replace this by a single $\left(a^{\perp} \otimes a\right)$ and remove the cut. For a cut $1 \Phi \perp$, the situation is analogous. Finally, a cut $[A \ngtr B] \oplus(C \otimes D)$ is simply replaced by two smaller cuts $A \oplus C$ and $B \oplus D$.

Let us now consider the cuts that involve the quantifiers. There are three cases, one for each of $\exists, \exists$, and $\exists$. If we have a cut $\exists a . A \oplus \forall a . B$, then let $C$ be a node with $\exists a . A \stackrel{\sigma}{\sim} C$ or $\exists a . A \stackrel{\sigma}{\curvearrowleft} C$. In the one case let $D=\lfloor C \triangleleft \sigma\rfloor$, in the other let $D=\lfloor C \triangleleft \sigma\rfloor^{\perp}$. By 5.71] $D$ is independent from the choice of $C$. If there are no stretching edges, then let $D=a$. Now we substitute inside the box of $\forall a . B$ the variable $a$ with $D$ and remove all doors of the $\forall$-box, and replace the cut by $A \oplus B$. Note that "removing a door" means for a 引 that the node is removed, but for an $\exists$ it means that the node is replaced by an $\exists$ and a stretching edge is added to/from every $D$ (resp. $D^{\perp}$ ) substituted for $a$ (resp. $a^{\perp}$ ) formerly bound by the $\exists$-node. Note also that by substituting $a$ with $D$ we get "axiom links" which are not atomic anymore, but it is straightforward to make them atomic again.

If we have a cut $\Xi a . A \oplus B$, let $e B$ be the empire of $B$, i.e, largest sub-proof graph of $P \stackrel{\nu}{\triangleright} \Gamma \triangleleft \sigma$ that has $B$ as a door, and let $B_{1}, \ldots, B_{n}$ be the other doors of $e B$ inside $\Gamma$, and let $R$ be the door of $e B$ in $P$. If $e B$ has more than one root-node inside the linking $P$, then we can rearrange the 8 -nodes in $P$ via our equivalence such that $e B$ has a single 8 -root in $P$. Furthermore, we can get in $P$ a subformula $[\exists a . Q \& R]$ where $\exists a . Q$ is the partner of $\Xi a$. $A$. We replace this by $\exists a .[Q \ngtr R]$, add an additional $\exists a$-node to each of $B_{1}, \ldots, B_{n}$, and reduce the cut to $\Xi a .(A \oplus B)$. Hence, we have pulled the whole empire of $B$ inside the box of $\Xi a . A$.

Finally, if we have a cut $\exists a . A \oplus \forall a . B$, then we simply merge the two boxes into one.

This cut reduction relation is defined a priori only on pre-proof graphs. But we have immediately the following:

7.1 Theorem The cut reduction relation preserves correctness and is well-defined on proof nets.
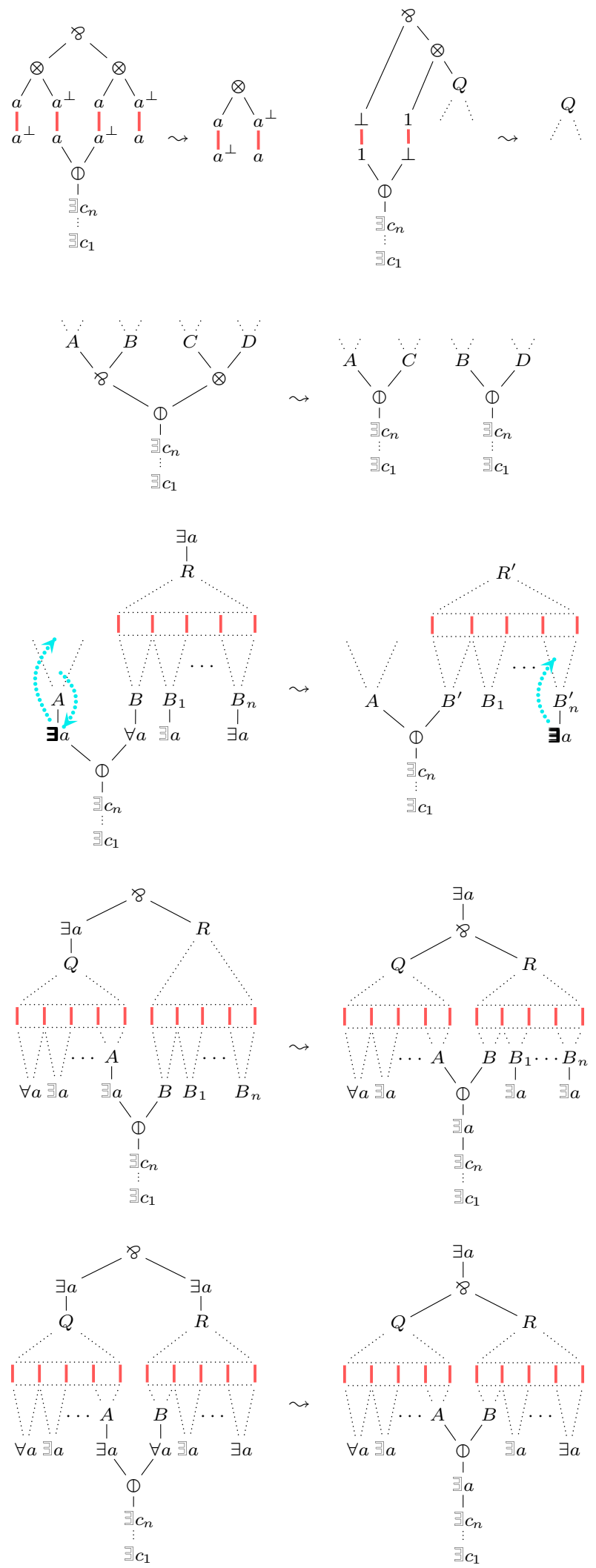

Figure 9. Cut reduction for MLL2 proof nets 
Our main results is now:

7.2 Theorem The cut reduction relation $\leadsto$ is terminating and confluent on proof nets.

An important consequence is that we have a category of proof nets: the objects are (simple) formulas and a map $A \rightarrow B$ is a proof net with conclusion $\vdash A^{\perp}, B$. The composition of maps is defined by cut elimination. Unfortunately, we do not know much about this category, apart from the fact that it is $*$-autonomous [17]. But there are some observations that we can make about the units, which can be expressed with the second-order quantifiers:

$$
1 \equiv \forall a \cdot\left[a^{\perp} 8 a\right] \quad \text { and } \quad \perp \equiv \exists a \cdot\left(a \otimes a^{\perp}\right)
$$

An interesting question to ask is whether these logical equivalences should be isomorphisms in the categorification of the logic. In the category of coherent spaces [10] they are, but in our category of proof nets they are not. This can be shown as follows. The two canonical maps $\forall a \cdot\left[a^{\perp} \varnothing a\right] \rightarrow 1$ and $1 \rightarrow \forall a \cdot\left[a^{\perp} \varnothing a\right]$ are given by:

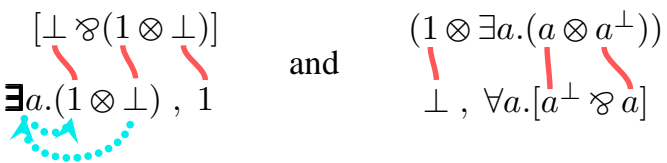

respectively. Composing them means eliminating the cut from

This yields

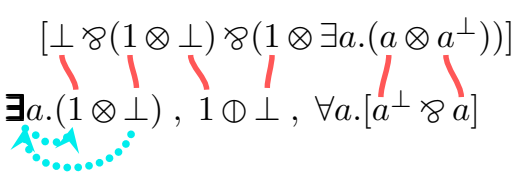

$$
\begin{aligned}
& {\left[\perp 8\left(1 \otimes \exists a \cdot\left(a \otimes a^{\perp}\right)\right)\right]} \\
& \exists a \cdot(1 \otimes \perp), \forall a \cdot\left[a^{\perp} \varnothing a\right] \\
& \wedge \circ \perp \ldots \bullet^{\circ}
\end{aligned}
$$

If the two maps in (10) where isos, the result (12) must be the same as the identity map $\forall a \cdot\left[a^{\perp} 8 a\right] \rightarrow \forall a \cdot\left[a^{\perp} 8 a\right]$ which is represented by the proof net

$$
\begin{aligned}
& \exists a .\left[\left(a^{\perp} \otimes a\right) 8\left(a \otimes a^{\perp}\right)\right] \\
& \exists a .\left(a \otimes a^{\perp}\right), \forall a .\left[a^{\perp} 8 a\right]
\end{aligned}
$$

This is obviously not the case (even if we replaced $\exists a$ by $\exists a . \exists a$ as for Theorem 6.2. A similar situation occurs with the additive units, for which we have

$$
0 \equiv \forall a . a \quad \text { and } \quad \top \equiv \exists a . a
$$

Since we do not have 0 and $\top$ in the language, we cannot check whether we have these isos in our category. However, since 0 and $T$ are commonly understood as initial and terminal objects of the category of proofs, we could ask whether $\forall a . a$ and $\exists a$. $a$ have this property: We clearly have a canonical proof for $\forall a . a \rightarrow A$ for every formula $A$, but it is $n o t$ necessarily unique.

However, the correct treatment of additive units in proof nets is still an open problem for future research.

\section{References}

[1] M. Abadi, L. Cardelli, P.-L. Curien, and J.-J. Lévy. Explicit substitutions. Journal of Functional Programming, 1(4):375-416, 1991.

[2] G. Bellin and J. van de Wiele. Subnets of proof-nets in MLL $^{-}$. In Advances in Linear Logic, pages 249-270. Cambridge University Press, 1995.

[3] R. Blute, R. Cockett, R. Seely, and T. Trimble. Natural deduction and coherence for weakly distributive categories. Journal of Pure and Applied Algebra, 113:229-296, 1996.

[4] K. Brünnler. Deep Inference and Symmetry for Classical Proofs. PhD thesis, Technische Universität Dresden, 2003.

[5] K. Brünnler and A. F. Tiu. A local system for classical logic. In LPAR 2001, volume 2250 of LNAI, pages 347-361. Springer, 2001.

[6] S. R. Buss. The undecidability of $k$-provability. Annals of Pure and Applied Logic, 53:72-102, 1991.

[7] V. Danos and L. Regnier. The structure of multiplicatives. Annals of Mathematical Logic, 28:181-203, 1989.

[8] N. G. de Bruijn. Lambda-calculus notation with nameless dummies, a tool for automatic formula manipulation. Indag. Math., 34:381-392, 1972.

[9] H. Devarajan, D. Hughes, G. Plotkin, and V. R. Pratt. Full completeness of the multiplicative linear logic of chu spaces. In (LICS 1999), 1999.

[10] J.-Y. Girard. Linear logic. Theoretical Computer Science, 50:1-102, 1987.

[11] A. Guglielmi. A system of interaction and structure. To appear in ACM Transactions on Computational Logic, 2002.

[12] A. Guglielmi and L. Straßburger. Non-commutativity and MELL in the calculus of structures. In L. Fribourg, editor, CSL 2001, volume 2142 of LNCS, pages 54-68. SpringerVerlag, 2001.

[13] D. Hughes. Simple free star-autonomous categories and full coherence. 2005.

[14] J.-B. Joinet. Completeness of MLL proof nets w.r.t. weak distributivity, 2006. To appear in Journal of Symbolic Logic.

[15] Y. Lafont and A. Scedrov. The undecidability of second order multiplicative linear logic. Information and Computation, 125:46-51, 1996.

[16] F. Lamarche and C. Retoré. Proof nets for the Lambekcalculus - an overview. In V. M. Abrusci and C. Casadio, editors, "Proofs and Linguistic Categories", pages 241262. CLUEB, Bologna, 1996.

[17] F. Lamarche and L. Straßburger. From proof nets to the free *-autonomous category. Logical Methods in Computer Science, 2(4:3):1-44, 2006.

[18] D. Miller. A compact representation of proofs. Studia Logica, 46(4):347-370, 1987.

[19] L. Straßburger. Linear Logic and Noncommutativity in the Calculus of Structures. PhD thesis, TU Dresden, 2003.

[20] L. Straßburger. From deep inference to proof nets. In Structures and Deduction - The Quest for the Essence of Proofs (Satellite Workshop of ICALP 2005), 2005.

[21] L. Straßburger and F. Lamarche. On proof nets for multiplicative linear logic with units. In CSL 2004, vol. 3210 of LNCS, pages 145-159. Springer, 2004. 